\title{
CONHECIMENTO E PRÁTICAS PREVENTIVAS RELACIONADAS ÀS DOENÇAS ONCOLÓGICAS DE MULHERES CLIMATÉRICAS
}

\author{
KNOWLEDGE AND PREVENTIVE PRACTICES RELATED TO ONCOLOGICAL
}

\author{
DISEASES OF CLIMATERIC WOMEN
}

\author{
Ana Paula Tiecker ${ }^{1}$ \\ Evelise Moraes Berlezi ${ }^{2}$ \\ Daiana Meggiolaro Gewehr ${ }^{3}$ \\ Vanessa Adelina Casali Bandeira ${ }^{4}$ \\ Recebido em: 05 dez. 2017 \\ Aceito em: 22 jun. 2018
}

RESUMO: Introdução: Em decorrência da transição epidemiológica brasileira, o câncer atualmente é um problema de saúde pública, pela sua elevada morbimortalidade. Objetivo: Verificar o conhecimento e práticas preventivas relacionadas às doenças oncológicas em mulheres climatéricas. Materiais e métodos: A pesquisa seguiu um delineamento transversal analítico, realizado com mulheres de idade entre 35 e 65 anos com cadastro ativo nas unidades de Estratégia Saúde da Família da área urbana de um município de médio porte da região noroeste do estado do Rio Grande do Sul/Brasil. Foram selecionadas 197 mulheres do banco de dados da pesquisa "Envelhecimento Feminino"; que responderam ao questionário. Resultados: A média de idade das mulheres foi $51,22 \pm 7,71$ anos. Observou-se que a renda familiar e a escolaridade têm associação com nível de conhecimento do câncer e suas causas. Sobre os fatores que causam o câncer $85,8 \%$ (169) relataram saber pelo menos uma causa; $34,5 \%$ (68) afirmaram que a equipe de saúde aborda o tema câncer; $69,5 \%$ (137) fazem com regularidade o autoexame de mama e $96,4 \%$ (190) já realizaram em algum momento o Papanicolau. Discussão: O conhecimento sobre o câncer e seus exames de detecção precoce tem relação diretamente proporcional com a motivação em relação ao cuidado em saúde. Conclusão: Os achados do estudo mostram que os usuários têm informação acerca do que é câncer e seus fatores de risco; porém é necessário que os profissionais de saúde trabalhem para que a informação se transforme em conhecimento capaz de mudar atitudes e comportamentos dos usuários.

Palavras-chave: Atenção Primária a Saúde. Envelhecimento. Diagnóstico precoce. Neoplasias.

\footnotetext{
1 Graduanda do curso de Fisioterapia da Universidade Regional do Noroeste do Estado do Rio Grande do Sul - UNIJUI. anapaulatiecker@hotmail.com.

2 Doutora em Gerontologia Biomédica, docente do Departamento de Ciências da Vida da Universidade Regional do Noroeste do Estado do Rio Grande do Sul - UNIJUI. Docente do corpo permanente do Programa de Pós-Graduação em Atenção Integral à Saúde UNIJUÍ/UNICRUZ e do Programa de Pós-Graduação da Universidade Federal de Santa Maria. Coordenadora da Pesquisa Envelhecimento Feminino. evelise@unijui.edu.br.

${ }^{3}$ Farmacêutica, Mestranda do Programa de Pós-Graduação em Atenção Integral à Saúde da Universidade Regional do Noroeste do Estado do Rio Grande do Sul - UNIJUI e Universidade de Cruz Alta - UNICRUZ. daiagewehr@hotmail.com.

${ }^{4}$ Farmacêutica, Mestre em Atenção Integral à Saúde da Universidade Regional do Noroeste do Estado do Rio Grande do Sul - UNIJUI e Universidade de Cruz Alta - UNICRUZ. vanessa.acbandeira@yahoo.com.br. Autor Correspondente: Evelise Moraes Berlezi. Departamento de Ciências da Saúde; Rua do Comércio, 3000; Bairro Universitário; ljuí- RS; CEP 98700 000. E-mail evelise@unijui.edu.br.
} 
ABSTRACT: Introduction: As a result of the Brazilian epidemiological transition, cancer is currently a public health problem due to its high morbidity and mortality. Objective: To verify the knowledge and preventive practices related to oncological diseases in climacteric women attached to the Family Health Strategy (FHS). Method: The research followed an analytical cross-sectional design. The population was consituted of women aged between 35 and 65 years with an active registry in the Family Health Strategy units of the urban area of a medium-sized municipality in the northwest region of the state of Rio Grande do Sul, Brazil. A total of 197 women were selected from the database of the research "Female Aging", who answered the questionnaire. Results and discussion: The average age of the women was $51.22 \pm$ 7.71 years. It was observed that family income and schooling have an association with the level of knowledge about cancer and its causes. Regarding the factors that cause cancer, $85.8 \%$ (169) reported knowing at least one cause; $34.5 \%(68)$ stated that the health team addresses the issue of cancer; $69.5 \%$ (137) regularly performed breast self-exam and $96.4 \%$ (190) had already performed the Papanicolaou test at some point. Knowledge about cancer and its early detection exams is directly proportional to motivation in relation to health care. Conclusion: The findings of the study show that users have information about cancer and its risk factors; however, it is necessary that health professionals work so that information becomes knowledge capable of changing attitudes and behaviors of users.

Keywords: Primary Health Care. Aging. Early Diagnosis. Neoplasms.

\section{INTRODUÇÃO}

Em decorrência da transição epidemiológica brasileira, o câncer atualmente é um problema de saúde pública, pela sua elevada morbimortalidade. De acordo com o Instituto Nacional de Câncer (INCA), no Brasil, estima-se que no período de 2016 a 2017 serão diagnosticados 600 mil casos novos de câncer; e destes, mais de $50 \%$ acometeram o sexo feminino. $O$ câncer de mama é o tipo de câncer que mais acomete as mulheres em todo o mundo. Esse tipo de câncer corresponde a $25 \%$ dos casos novos por ano. Ao observar a distribuição dessa doença no território brasileiro verifica-se que as maiores taxas de incidência e mortalidade ocorrem nas Regiões Sul e Sudeste e as menores nas Regiões Norte e Nordeste (INCA, 2014a; INCA, 2016).

O câncer é uma doença multifatorial e complexa que resulta da interação entre fatores genéticos, ambientais e estilo de vida. Neste contexto, há fatores de risco não modificáveis como história familiar, idade, sexo; e fatores modificáveis, que são responsáveis por mais de um terço das mortes por câncer; estes estão relacionados a alimentação, especialmente, com baixo consumo de frutas, legumes e verduras; a inatividade física; sobrepeso, obesidade e poluição (INCA, 2014b).

Além disso, a ocorrência de câncer está diretamente relacionada ao aumento da população idosa (INCA, 2016). O aumento desse tipo de doença na população geriátrica está associado, para além, da interação genética, ambiental e estilo de vida; ao próprio processo de senescência. Sabe-se que à medida que o organismo envelhece mecanismos protetores que identificam a transcrição de erros genéticos no processo de replicação 
celular são menos eficientes; em organismos jovens há um potencial maior de identificação de erros de transcrição e quando evidenciado induz a morte celular programada (ITAHANA; DIMRI; CAMPISI, 2001).

Frente a esse problema de saúde pública a prevenção, pautada essencialmente, na adoção de um estilo de vida ativo e alimentação saudável parece ser o caminho para reduzir a incidência e mortalidade. Nesse contexto, ações de educação que estimulem o cuidado com a saúde e a adoção de comportamento preventivo são fundamentais para a detecção precoce e controle da doença. $O$ câncer quando detectado em sua fase inicial tem maior probabilidade de cura e baixa morbidade decorrente do tratamento, ou seja, a detecção e tratamento precoce reduzem a chance de complicações e sequelas durante e pós-tratamento; além de reduzir o risco de metástases e mortalidade (GOMES, 2016).

No contexto das doenças oncológicas os exames preventivos periódicos são a forma mais eficiente de detectar lesões precursoras e fazer diagnóstico (INCA, 2017). Dados do Ministério da Saúde estimam que $40 \%$ das mulheres brasileiras nunca fizeram o exame Papanicolau, que detecta lesões precursoras de câncer de colo de útero e que é realizado nas unidades básicas de saúde (BRASIL, 2002).

Há evidencias científicas que fatores como idade avançada, baixo nível socioeconômico, pertencer à raça negra ou parda e ser solteira associa-se a grupos populacionais que não realizam exames preventivos; além disso, questões culturais como o receio da dor, vergonha, desconhecimento do procedimento e do local de realização (BRISCHILIARI et al., 2012; LUCENA et al., 2011) também interferem na realização de exames preventivos. Conforme Rutledge et al. (2001) o aumento do conhecimento sobre o câncer de mama e de seus exames de detecção precoce aumentam a motivação em relação à saúde, podendo influenciar a prática da realização desses exames.

Considerando a heterogeneidade social, econômica, cultural, ambiental da população brasileira tomando como base as regiões federativas e, também, as diferenças dentro do território estadual justifica-se a proposição de diagnósticos populacionais do microterritório para que se conheça a realidade, as particularidades e as condições de saúde das populações. O diagnóstico populacional é o substrato para o planejamento e gestão do cuidado de indivíduos e populações de forma racionalizada e em todos os níveis de atenção; na perspectiva de maior resolutividade dos problemas de saúde. Neste contexto, o objetivo do estudo foi verificar o conhecimento e práticas preventivas relacionadas às doenças oncológicas em mulheres climatéricas adstritas a Estratégia Saúde da Família.

\section{MATERIAIS E MÉTODOS}

Estudo de delineamento transversal, analítico realizado por meio do acesso ao banco de dados da pesquisa institucional "Envelhecimento Feminino" da Universidade Regional do Noroeste do Estado do Rio Grande do Sul (UNIJUí), aprovada pelo Comitê de 
Ética e Pesquisa, sob o Parecer Consubstanciado n²94.456/2014.

A população da pesquisa foi constituída por mulheres, com idade entre 35 e 65 anos, período que corresponde ao climatério, adstritas às unidades de Estratégia Saúde da Família (ESF) da área urbana de um município de médio porte da região noroeste do estado do Rio Grande do Sul/Brasil. Para o presente estudo, foram selecionadas 197 mulheres do banco de dados; o critério de seleção foi terem respondido ao questionário sobre as características sociodemográficas e do conhecimento acerca de fatores de risco e comportamento preventivo para doenças oncológicas. As informações que compõem o banco de dados foram coletados por meio de inquérito domiciliar pelas acadêmicas e docentes integrantes do projeto de pesquisa supracitado.

As variáveis de interesse foram: dados sociodemográficos: idade, escolaridade, renda familiar e estado civil; e questões referentes ao conhecimento das doenças oncológicas: Você tem conhecimento sobre o que é câncer?; Você saberia dizer 3 causas de câncer?; De onde você tem este conhecimento sobre as causas de câncer; A equipe de saúde alguma vez já abordou este tema (câncer) com você?; Se este tema foi abordado em que situação isso ocorreu?; Você se considera uma pessoa que cuida da saúde como: cuidados com alimentação prática de atividade física, atividades para controle do stress entre outros?; Você com regularidade marca e comparece a consultas médicas ou de enfermagem para realizar exames?; Você foi ensinada pela equipe de saúde (médico/enfermeira/técnica de enfermagens/agentes comunitários) a fazer o autoexame da mama?; Você faz com regularidade o autoexame da mama?; Você sabe o que é o exame Papanicolau (o preventivo)?; Você já realizou o exame Papanicolau?; Quantas vezes já fez este exame?; Faz pelo menos uma vez no ano?; Onde realizou o exame Papanicolau?.

Para a análise dos dados foi utilizado o software Statistical Package for the Social Sciences (SPSS) (versão 20.0). Para variáveis quantitativas utilizaram-se medidas de tendência central, de dispersão e variabilidade; e para as variáveis qualitativas, frequências relativa e absoluta. Para testar a hipóteses de associações de duas variáveis utilizou-se o teste de qui-quadrado de Pearson com confiabilidade de $95 \%$.

\section{RESULTADOS E DISCUSSÃo}

Foram incluídas no estudo 197 mulheres, com média de idade 51,22 $\pm 7,71$ (IC 95\% $50,14-52,30)$ anos. A maioria relatou renda igual ou superior a dois salários mínimos $(45,6 \%)$; que residiam com companheiros $(68,0 \%)$ e tinham baixa escolaridade $(50,8 \%)$; ensino fundamental ou analfabetas funcionais.

A Tabela 1 mostra a associação entre as variáveis sociodemográficas com o conhecimento do que é câncer e os seus fatores de risco. Ao analisar a relação dessas variáveis os resultados sugerem que a renda familiar e a escolaridade possam ter influência sobre o nível de conhecimento da doença e suas causas. 
Tabela 1: Associação das variáveis sociodemográficas com o conhecimento sobre o que é câncer e suas causas, ljuí-RS, 2017.

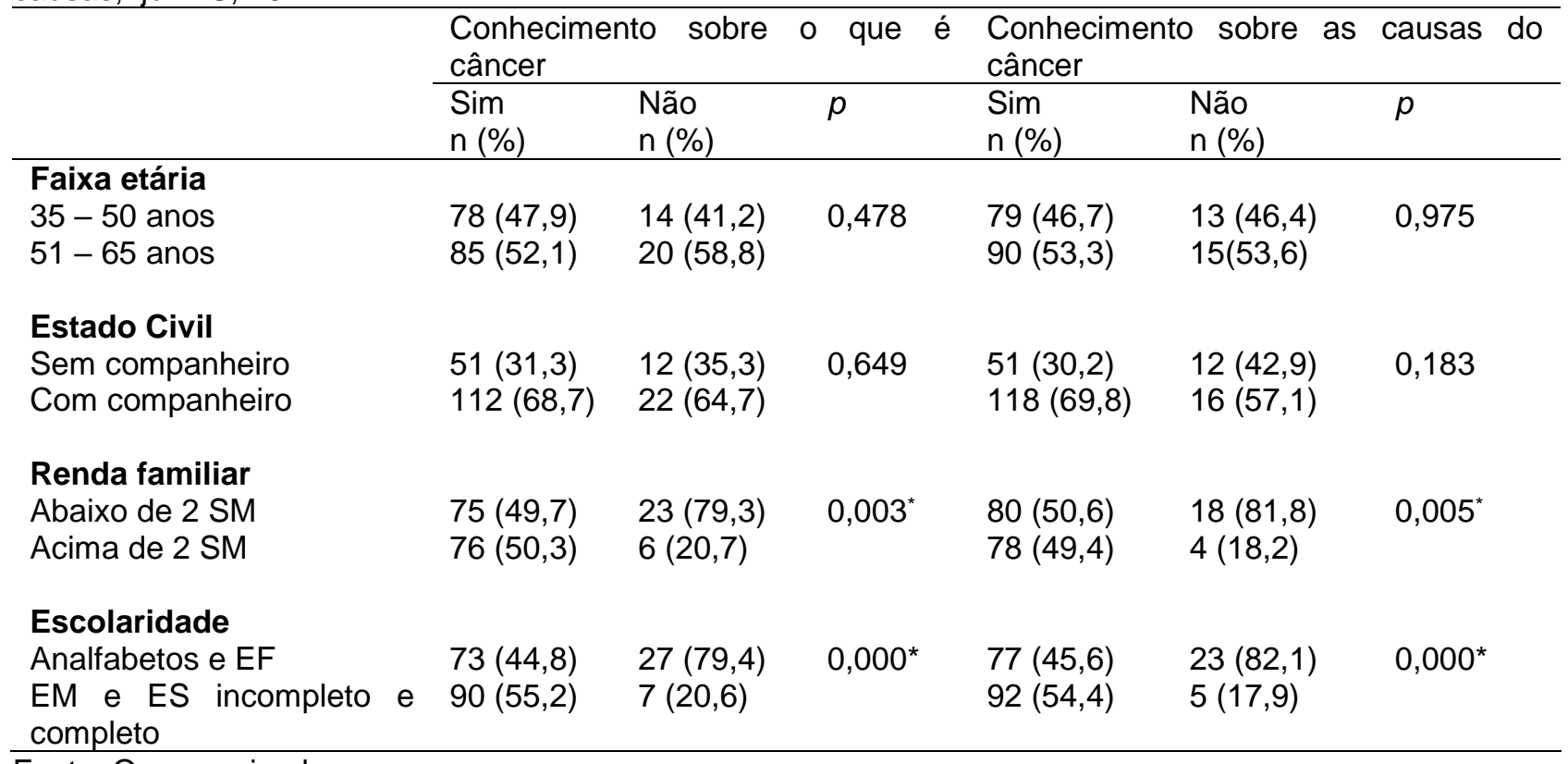

Fonte: Os pesquisadores

${ }^{*} \mathrm{p}<0,005$.

SM: Salário Mínimo; EF: Ensino fundamental; EM: Ensino médio; ES: Ensino Superior.

Em relação à ocorrência das doenças oncológicas prévias 8,6\% (16) das mulheres relataram o diagnóstico de câncer. O câncer de mama foi o mais prevalente, com ocorrência de $31,25 \%$ (5), seguido de câncer do colo do útero 18,75\% (3), 12,5\% (2) câncer de tireoide, 12,5\% (2) câncer de pele, 6,25\% (1) câncer renal, 6,25\% (1) câncer abdominal e 6,25\% (1) não soube responder qual o tipo de câncer. A baixa escolaridade e a baixa renda constituem-se em barreiras para o acesso de informações pertinentes e de boa qualidade (BONOTTO; MENDOZA-SASSI; SUSIN, 2016).

No presente estudo, observou-se associação entre escolaridade e conhecimento das mulheres em relação às doenças oncológicas. A influência da escolaridade também foi evidenciada por outros estudos como o realizado por Leite et al. (2014) com mulheres entre 25 e 59 anos cadastradas nas unidades de saúde de um bairro de Bauru-SP, o qual verificou que as mulheres com escolaridade superior a sete anos apresentaram maior conhecimento acerca do câncer de colo de útero. Assim como, Batiston et al. (2011) em estudo realizado com mulheres de 40 a 69 anos de Dourados-MS verificaram que o conhecimento quanto ao câncer de mama e seus fatores de risco é maior a medida que o nível de escolaridade aumenta.

Ainda, para além do conhecimento sobre a doença o estudo Batiston et al. (2011) apontou que a renda, idade e escolaridade influenciam sobre os exames preventivos como a mamografia; destacando a importância de projetos informativos e educacionais que respeitem as necessidades e limitações de cada mulher, com vistas a proporcionar a divulgação e compreensão das informações independente a renda ou escolaridade.

Quando questionadas sobre os fatores que causam o câncer, 85,8\% (169) das 
mulheres relataram saber pelo menos uma causa. Foram descritas um total de 456 causas, considerando a diversidade de nomeações dos fatores, as mais citadas foram: tabagismo por 75,15\% (127) das mulheres, alimentação inadequada 50,89\% (86), uso de bebidas alcoólicas 46,75\% (79), hereditariedade 35 (20,71\%), estresse 25 (14,79\%), agrotóxico 12,43 (21) e exposição ao sol 8,88\% (15).

Ao inquerir as mulheres sobre se a equipe de saúde já abordou o tema câncer $34,5 \%$ (68) mulheres responderam que sim; dessas 58,82\% (40) referiram ter sido abordada durante as consultas médicas, 16,18\% (11) durante a visita do agente comunitário de saúde, 16,18\% (11) em reunião de grupo de saúde e 17,65\% (12) em outras situações.

Quando questionadas quanto à forma de obtenção desses conhecimentos, os mais citados foram: Meios de comunicação por 45,68\% (90) mulheres, com amigos e familiares $16,75 \%$ (33), por experiência própria ou de familiares $12,18 \%$ (24) e conversas em grupos $11,67 \%$ (23). Em relação ao comportamento preventivo $85,3 \%$ (168) das mulheres afirmaram que marcam e comparecem com regularidade as consultas médicas ou de enfermagem para realizar exames.

Com relação aos exames preventivos Papanicolau e autoexame da mama identificou-se que $89,3 \%$ (176) das mulheres relataram que a equipe da ESF ensinou 0 procedimento para realizar o autoexame das mamas e dessas 69,5\% (137) fazem com regularidade o autoexame. No que se refere à realização Papanicolau 93,4\% (184) das mulheres têm conhecimento sobre o que é o exame e $96,4 \%$ (190) já realizaram em algum momento; além disso, 77,7\% (153) fazem o exame pelo menos uma vez ao ano. Em relação ao local de realização 70,1\% (138) realizam no Sistema Único de Saúde (SUS) e 25,4\% (50) realiza em consultório médico por plano privado. Não foi observada associação entre as variáveis sociodemográficas e a regularidade de realização do exame Papanicolau e o autoexame da mama (Tabela 2).

Tabela 2: Associação das variáveis sóciodemograficas com a realização de exames preventivos com em mulheres no climatério, ljuí-RS, 2017.

\begin{tabular}{|c|c|c|c|c|c|c|}
\hline & \multicolumn{3}{|c|}{$\begin{array}{l}\text { Você faz regularmente } 0 \\
\text { exame Papanicolau? }\end{array}$} & \multicolumn{3}{|c|}{$\begin{array}{l}0 \text { autoexame da } \\
\text { mama? }\end{array}$} \\
\hline & $\begin{array}{l}\text { n (\%) } \\
\text { Sim }\end{array}$ & $\begin{array}{l}\text { n (\%) } \\
\text { Não }\end{array}$ & $p$ & $\begin{array}{l}\text { n (\%) } \\
\text { Sim }\end{array}$ & $\begin{array}{l}\text { N (\%) } \\
\text { Não }\end{array}$ & $p$ \\
\hline $35-50$ anos & $72(47,1)$ & $20(45,5)$ & 0,851 & $64(46,7)$ & $27(46,6)$ & 0,983 \\
\hline $51-65$ anos & $81(52,9)$ & $24(54,5)$ & & $73(53,3)$ & $31(53,4)$ & \\
\hline \multicolumn{7}{|l|}{ Estado Civil } \\
\hline Solteira/sem companheiro & $46(30,1)$ & $17(38,6)$ & 0,283 & $45(32,8)$ & $17(29,3)$ & 0,628 \\
\hline Com companheiro & $107(69,9)$ & $27(61,4)$ & & $92(67,2)$ & $41(70,7)$ & \\
\hline \multicolumn{7}{|l|}{ Renda familiar } \\
\hline Abaixo de 2 SM & $93(53,7)$ & $5(71,4)$ & & $65(52,0)$ & $32(60,4)$ & 0,305 \\
\hline Acima de 2 SM & $80(46,3)$ & $2(28,6)$ & & $60(48,0)$ & $21(39,6)$ & \\
\hline \multicolumn{7}{|l|}{ Escolaridade } \\
\hline Analfabetos e EF & $98(51,6)$ & $2(28,6)$ & & $67(48,9)$ & $32(55,2)$ & \\
\hline $\begin{array}{l}\text { EM e ES incompleto e } \\
\text { completo }\end{array}$ & $92(48,4)$ & $5(71,4)$ & & $70(51,1)$ & $26(44,8)$ & \\
\hline
\end{tabular}

SM: Salário Mínimo; EF: Ensino fundamental; EM: Ensino médio; ES: Ensino Superior. 
É possível observar nesse estudo que as informações sobre os fatores de risco para o câncer não foram ofertadas por profissionais de saúde, mas outras fontes que não estão diretamente ligadas à área da saúde. Ao encontro desse achado, o estudo de Rodrigues et al. (2016), verificou que as mulheres adquiriram conhecimento sobre 0 autoexame das mamas principalmente através da mídia leiga, sendo um fato que merece atenção, visto que a disseminação de informações algumas vezes com baixo rigor cientifico podem não ser apropriado.

Como limitação desse estudo, infere-se que as mulheres podem ter recebido informações sobre o câncer e seus fatores de risco pela equipe de saúde, no entanto, podem não ter compreendido essas informações ou não recordaram durante a coleta de dados. Cabe destacar que muitas vezes o tema é abordado, mas merece ter diversas estratégias e ações de educação em saúde para que a população tenha maior compreensão e, dessa forma, adoção de comportamento preventivo. Por isso, é de suma importância que se desenvolvam metodologias pelas equipes de saúde que avaliem o nível de compreensão dos usuários das informações prestadas pelos profissionais.

Cabem as equipes de saúde prestar cuidado integral a população através de ações de promoção de saúde, rastreamento e detecção precoce; mas somente com a participação ativa do usuário é que se tem em perspectiva a mudança do estilo de vida e a adoção de comportamento e atitudes que privilegiam a saúde plena. Sobre isso, o estudo de Rutledgeet, et al. (2001) destacou que o conhecimento sobre o câncer de mama e seus exames de detecção precoce tem relação diretamente proporcional com a motivação em relação ao cuidado em saúde, mostrando que quanto maior o conhecimento maior será a motivação, podendo influenciar na prática e realização dos exames. Ainda, os estudos de Silva et al. (2011) e Oliveira et al. (2012) constataram que estratégias de educação em saúde são de grande importância no contexto oncológico de prevenção do câncer, pois levam a comportamentos mais saudáveis, maior adesão a práticas preventivas, além de retirar medos e estigmas.

Quando questionadas sobre os fatores que causam o câncer, as mulheres mostraram saber pelo menos uma causa, as quais estavam de acordo com os elencados pelo INCA (2016), ponto que se mostra como uma característica positiva na população estudada. Hábitos de vida não saudáveis como fumar, ingerir bebida alcoólica, sedentarismo e obesidade, entre outros, não aumentam somente a probabilidade do surgimento de câncer, mas também de outros tipos de cânceres e diversas outras doenças crônico-degenerativas (MATOS; PELLOSO; CARVALHO, 2010). A modificação no estilo de vida da população, destacando a tendência global à predominância de estilos de vida não saudáveis contribui consideravelmente para o aumento das taxas de incidência do câncer. Sendo assim, os fatores de risco modificáveis devem ser estudados e trabalhados com a população a fim de possibilitar a busca por um estilo de vida saudável e prevenir o câncer.

Em relação à realização dos exames preventivos a maioria das mulheres referiram conhecer os exames e realiza-los, independente da renda ou escolaridade (Tabela 2), o que é positivo para a detecção precoce. Sabe-se que o câncer de mama e colo do útero 
são os mais frequentes o que também foi evidenciado nesse estudo, na maioria das vezes esses tipos de câncer são possíveis de serem diagnosticados precocemente em fase inicial através de exames como o Papanicolau, mamografia e o autoexame da mama que é utilizado como estratégia secundária (INCA, 2016).

O rastreamento precoce mostra-se como uma estratégia eficaz na redução das mortes principalmente em mulheres acima de 50 anos (INCA, 2016). Não obstante de reconhecer que a organização dos serviços e a rede de saúde do SUS têm gargalos que dificultam o acesso aos exames preventivos; também, há pelo viés do usuário a adoção de um comportamento de buscar os serviços de saúde somente quando apresentam sinais ou sintomas de que algo não vai bem. Ainda, há um grande desconhecimento da população da importância e repercussões da detecção precoce da doença; além disso, o sentimento de medo de um possível diagnóstico muitas vezes impede a busca ao serviço de saúde (GOMES, 2016; OSHIRO et al., 2014). Essas questões que podem levar, ao diagnóstico tardio explica, em partes, o porquê as taxas de mortalidade se mantêm altas mesmo com maior aparato tecnológico.

Em estudo desenvolvido por Oshiro et al. (2014), com objetivo de analisar os eventos relacionados ao diagnóstico em estádio avançado do câncer de mama verificaram a trajetória percorrida pelas mulheres nos serviços de saúde; os resultados mostraram que a desinformação sobre a doença, a demora na procura por atendimento no serviço de saúde após a detecção da alteração mamária foram fatores que contribuíram para o diagnóstico da doença em estágio avançado. Já em estudo realizado por Mendonça et al. (2011) que objetivou analisar a compreensão de enfermeiros e usuárias da atenção primária sobre a adesão da prevenção ao câncer de colo uterino, os enfermeiros apontaram deficiência na organização, do suprimento de insumos e da manutenção de materiais na atenção primária, como fatores que dificultavam a realização do exame, enquanto que para as usuárias, a vergonha, medo, nervosismo e até mesmo pelo fato de ser um profissional do sexo masculino que realizava esse exame, eram variáveis que retardavam a procura pelo atendimento.

A ação educativa com respeito mútuo entre paciente e profissional de saúde é um importante passo para que a mulher compreenda a importância do exame preventivo e sinta-se motivada a realizá-lo. Esse relacionamento interpessoal, por meio de uma comunicação efetiva, é primordial para que a mulher tenha confiança no profissional de saúde e supere as eventuais dificuldades (SANTOS; CHUBACI, 2011).

Para que o investimento em ações de promoção da saúde e prevenção de doenças tenham efetividade e atinjam os objetivos esperados com repercussões futuras é necessário haver mudanças de paradigmas do modelo de saúde, especialmente no que diz respeito às doenças oncológicas. É consenso que, para acontecer essa mudança, há a necessidade de investimento na formação de profissionais de saúde comprometidos e preparados para atuar na educação em saúde, assim como nos diferentes ciclos de vida, com os diversos determinantes do processo saúde- -doença, com abordagem integral do sujeito nos muitos pontos da rede de atenção à saúde (HERR et al., 2013). 
Além disso, há a necessidade da participação dos usuários do sistema de saúde nas ações desenvolvidas no campo da educação em saúde, para que promovam o autocuidado e façam a opção por práticas que gerem a sua saúde. Somente o somatório de práticas dos profissionais e dos usuários pode repercutir, positivamente, nos indicadores de saúde.

Estes achados tornam-se importantes para propor e/ou reforçar políticas e programas já existentes, mas sobretudo utilizar para o planejamento das ações de saúde, especialmente na atenção primária, porta de entrada do SUS, tendo em vista as estatísticas progressivas da doença, que nos mostram que este processo de educação em saúde não está acontecendo.

\section{CONCLUSÕES}

Este estudo teve como objetivo verificar o conhecimento e práticas preventivas relacionadas às doenças oncológicas. Foi evidenciado que as mulheres com menor escolaridade e menor renda têm menor conhecimento sobre o câncer e seus fatores de risco; e, que há informação dos usuários acerca da prevenção do câncer.

Frente as estimativas para os próximos anos do número de casos novos de câncer os profissionais de saúde, especialmente das Equipes das Estratégias Saúde da Família, têm como o desafio atuarem de forma contundente na promoção da saúde e prevenção de doenças; e para isso, precisam encontrar os caminhos para que a informação se transforme em conhecimento capaz de mudar atitudes e comportamentos dos usuários; para que estes sejam sujeitos ativos no processo de cuidado com a saúde.

As doenças não transmissíveis requerem a adoção de um estilo de vida saudável; e isso, só é possível, quando se alia consciência sobre a importância do cuidado com a saúde com conhecimento acerca de fatores que interferem no bem-estar do indivíduo gerando doença.

Neste contexto, a educação é entendida como um processo de ensino e aprendizagem em que profissional e usuário estabelecem uma relação dialógica; em que a informação é contextualizada com a realidade socioeconômica e cultural, para que seja entendida e tenha significado; e se traduza em mudança de postura e comportamento adotando hábitos de vida saudável e dessa forma reduzindo o risco de adoecimento.

\section{REFERÊNCIAS}

BATISTON, A. P. et al. Conhecimento e prática sobre os fatores de risco para o câncer de mama entre mulheres de 40 a 69 anos. Revista Brasileira Saúde Materno Infantil, v. 11, n. 2, p. 163-171, 2011.

BONOTTO, G. M.; MENDOZA-SASSI, R. A.; SUSIN, L. R. O. Conhecimento dos fatores 
de risco modificáveis para doença cardiovascular entre mulheres e seus fatores associados: um estudo de base populacional. Revista Ciência \& Saúde Coletiva, v. 21, n. 1, p. 293-302, 2016.

BRASIL, Ministério da Saúde. Secretaria de Assistência à Saúde. Prevenção do câncer do colo do útero: manual técnico - profissionais de saúde. Brasília: 2002.

BRISCHILIARI, S. C. R. et al. Papanicolaou na pós-menopausa: fatores associados a sua não realização. Cadernos de Saúde Pública, v. 28, n. 10, p. 1976-1984, 2012.

GOMES, A. M. F. Conhecimento, atitude e prática de mulheres com câncer de mama sobre os métodos de detecção precoce. Defesa 20 de jul. 2016. F.10 Dissertação (Mestrado em Enfermagem) - Faculdade de Farmácia, Odontologia e Enfermagem. Universidade Federal do Ceara. Fortaleza, 2016.

HERR, G. E. et al. Avaliação de Conhecimentos acerca da Doença Oncológica e Práticas de Cuidado com a Saúde. Revista Brasileira de Cancerologia, v. 59, n. 1, p. 33-41, 2013.

INCA. Ministério da Saúde- Brasil. Secretaria de Atenção à Saúde. Instituto Nacional de Câncer. Coordenação de Prevenção e Vigilância de Câncer. Estimativa 2014: incidência de câncer no Brasil 2014 [on-line]. Rio de Janeiro (RJ): INCA; 2014a. Disponível em: http://www.inca.gov.br/bvscontrolecancer/publicacoes/Estimativa_2014.pdf. [acesso 07 de jun de 2017].

INCA. Ministério da Saúde- Brasil. Secretaria de Atenção à Saúde. Instituto Nacional de Câncer. Coordenação de Prevenção e Vigilância de Câncer. Prevenção, fatores de risco. Rio de Janeiro (RJ): INCA; 2014b [on-line]. Disponível em:

http://www2.inca.gov.br/wps/wcm/connect/cancer/site/prevencao-fatores-de-risco/como prevenir-cancer [acceso 10 de jun de 2017].

INCA. Ministério da Saúde- Brasil. Secretaria de Atenção à Saúde. Instituto Nacional de Câncer. Coordenação de Prevenção e Vigilância de Câncer. Estimativa 2016: incidência de câncer no Brasil 2016 [on-line]. Rio de Janeiro (RJ): INCA; 2016. Disponível em: http://www.inca.gov.br/estimativa/2016/estimativa-2016-v11.pdf. [acesso 07 de jun de 2017].

INCA. Ministério da Saúde- Brasil. Secretaria de Atenção à Saúde. Instituto Nacional de Câncer. Coordenação de Prevenção e Vigilância de Câncer. Detecção Precoce. [online]. Rio de Janeiro (RJ): INCA; 2017. Disponível em:

<http://www2.inca.gov.br/wps/wcm/connect/tiposdecancer/site/home/mama/deteccao_prec oce++> [acceso 07 de jul de 2017].

ITAHANA, K.; DIMRI, G.; CAMPISI, J. Regulation of cellular senescence by p53. Eur. J. Biochem, v. 268, p. 2784-2791, 2001.

LEITE, M. F. et al. Conhecimentos E Prática Das Mulheres Sobre Câncer De Colo Do Útero de Uma Unidade Básica de Saúde. Journal of Human Growth and Development, v. 24, n. 2, p. 208-213, 2014.

LUCENA, L. T. et al. Fatores que influenciam a realização do exame preventivo do câncer cérvico-uterino em Porto Velho, Estado de Rondônia, Brasil. Rev Pan-Amaz Saúde, v. 
55, n. 69, p. 45-50, 2011.

MATOS, J. C. DE; PELLOSO, S. M.; CARVALHO, M. D. D. B. Prevalência de fatores de risco para o câncer de mama no município de Maringá, Paraná. Revista LatinoAmericano Enfermagem, v. 18, n. 3, p. 57-64, 2010.

MENDONÇA, F. A. DA C. et al. Prevenção Do Câncer De Colo Uterino: Adesão De Enfermeiros E Usuárias Da Atenção Primária. Revista Rene, v. 2, n. 12, p. 261-270, 2011.

OLIVEIRA, A. M. et al. Ações extensionistas voltadas para a prevenção e o tratamento do câncer ginecológico e de mama: relato de experiência. Revista da Escola de Enfermagem, v. 46, n. 1, p. 240-245, 2012.

OSHIRO, M. DE L. et al. Câncer de Mama Avançado como Evento Sentinela para Avaliação do Programa de Detecção Precoce do Câncer de Mama no Centro-Oeste do Brasil. Revista Brasileira de Cancerologia, v. 60, n. 1, p. 15-23, 2014.

RODRIGUES, T. C. G. F. et al. Conhecimento de 820 mulheres atendidas no Hospital Universitário da Universidade Federal de Juiz de Fora sobre autoexame das mamas.

Revista Brasileira de Mastologia, v. 26, n. 2, p. 60-64, 2016.

Rutledge DN, Barsevick A, Knobf MT, Bookbinder M. Breast cancer detection: knowledge, attitudes, and behaviors of women from Pennsylvania. Oncol Nurs Forum. V.28, n. 6, p. 1032-40, 2001.

SANTOS, G. D. DOS; CHUBACI, R. Y. S. O conhecimento sobre o câncer de mama e a mamografia das mulheres idosas frequentadoras de centros de convivência em São Paulo (SP, Brasil ). Ciência \& Saúde Coletiva, v. 16, n. 5, p. 2533-2540, 2011.

SILVA, P. A. DA; RIUL, S. DA S. Câncer de mama: fatores de risco e detecção precoce. Revista Brasileira de Enfermagem, v. 64, n. 6, p. 1016-21, 2011. 